\title{
Random matrices, random polynomials and Coulomb systems
}

\author{
P. Leboeuf \\ Laboratoire de Physique Théorique et Modèles Statistiques \\ Université de Paris-Sud, Bât.100 \\ 91405 Orsay Cedex, France
}

\begin{abstract}
It is well known that the joint probability density of the eigenvalues of Gaussian ensembles of random matrices may be interpreted as a Coulomb gas. We review these classical results for hermitian and complex random matrices, with special attention devoted to electrostatic analogies. We also discuss the joint probability density of the zeros of polynomials whose coefficients are complex Gaussian variables. This leads to a new two-dimensional solvable gas of interacting particles, with non-trivial interactions between particles.
\end{abstract}

To appear in the Proceedings of the International Conference on Strongly Coupled Coulomb Systems, Saint-Malo, 1999 (EDP Sciences, Les Ulis).

\section{INTRODUCTION}

The study of high lying states in the spectrum of atomic nuclei led to the introduction of a new type of statistical mechanics [1, 2]. Contrary to the traditional one, this new probabilistic theory does not associate a weight function to the different configurations a system may take, but rather introduces a statistical law directly at the level of the Hamiltonian. Depending on the symmetries of the system, an ensemble of Hamiltonians is defined with a proper probability distribution function. The statistical properties of the eigenvalues and eigenvectors of such random matrices (or averages of physical quantities over such distributions) may then be compared to a specific system, in which the average is made over some energy window.

Motivated by its mathematical tractability, one of the original ensembles introduced is the so-called Gaussian ensemble of random matrices, defined as the set of matrices whose elements are Gaussian independent variables. The probability density of a given $N \times N$ hermitian matrix is defined as

$$
P(H)=\mathcal{N} \exp \left(-\frac{\beta}{2} \operatorname{tr}\left(H^{2}\right)\right)
$$

where $\mathcal{N}$ is a normalization constant. A very specific aspect of this ensemble is its invariance under rotations in Hilbert space. The form of $H$ depends on symmetry considerations: its matrix elements are real for even spin systems with time reversal symmetry, complex when no symmetry is present and quaternion for odd spins with time reversal symmetry. For these three cases, the parameter $\beta$ in Eq.(1) takes the value 1, 2 and 4, respectively.

During the 40 years following its introduction, random matrix theory (RMT) has proven to be a pervasive theory (see e.g.[7]). Its applicability ranges from the description of atomic and molecular systems [3], the behavior of classically chaotic systems [4], the closely related motion of electrons in a disordered potential [5], up to the description of the statistical properties of the critical zeros of the Riemann zeta function [6]. In the second section of this paper our aim is to present a brief survey of some elementary results on the statistical properties of eigenvalues and 
eigenvectors associated to Eq.(1). The most characteristic feature concerning the distribution of eigenvalues is the existence of a strong repulsion acting among them. This repulsion may be seen, in an electrostatic interpretation, as a two-dimensional Coulomb interaction. For short distances, this Coulomb interaction is not a particular feature of the ensemble (1) but rather constitutes a very basic and general fact of any quantum mechanical system. The general arguments leading to this result from standard perturbation theory are also presented in section 2 .

Because of the symmetry of $H$, the eigenvalues lie on the real axis of the complex plane. A fully two-dimensional Coulomb gas arises when the basic hermitian symmetry of the Hamiltonian is abandoned. We illustrate this in section 3 with the example of the ensemble of complex matrices introduced by Ginibre [8]. We discuss in some detail the nearest neighbor spacing distribution in the complex plane since this quantity has been less studied in two dimensions.

In the same way the spectrum of random matrices may be associated to the equilibrium properties of one or two-dimensional Coulomb systems, it is possible to associate to the eigenstates of random matrices a two-dimensional gas of interacting particles (section 4). Although it contains a Coulomb repulsion, the full interaction between particles is now more complicated and includes many-body terms.

This reduction of the statistical properties of eigenstates to a two-dimensional interacting system is done through the Bargmann or coherent state representation of quantum mechanics. For finite (but large) matrices like the ones considered here, this representation associates to each eigenstate a polynomial whose coefficients, according to RMT, are random independent distributed. The reduction is completed when looking at the distribution of the roots in the complex plane of these random polynomials. The joint probability density of the zeros may be interpreted as a two-dimensional gas with the properties mentioned above.

The study of the distribution of roots of random polynomials has been an intensive field of research in recent years [9]-21]. For a Gaussian distribution of coefficients the problem has been solved. The density [10, 12] and all higher correlation functions [13] have been computed analytically for arbitrary variances. The gas associated to the zeros of random polynomials with Gaussian coefficients constitutes therefore a further example in the restricted class of twodimensional solvable models (at a certain temperature). Section 4 is a brief introduction and summary of some results on roots of random polynomials.

\section{RANDOM HERMITIAN MATRICES}

Consider the ensemble of $N \times N$ random hermitian matrices defined in Eq.(1). The basic problem to solve is to find the distribution function of the associated eigenvalues and eigenvectors. We denote $E_{\alpha}, \alpha=1, \ldots, N$ the eigenvalues and $\left\{p_{k}\right\}$ a parametrization of the eigenvectors of $H$ (the number of parameters $p_{k}$ depends on the symmetry of $H$ ). In the basis where $H$ is diagonal, $\operatorname{tr} H^{2}=\sum_{\alpha} E_{\alpha}^{2}$. Moreover, the Jacobian of the transformation is given by $[7$

$$
\mathcal{J}=\frac{\partial\left(H_{i j}\right)}{\partial\left(E_{\alpha}, p_{k}\right)}=\prod_{i<j=1}^{N}\left|E_{i}-E_{j}\right|^{\beta} .
$$

The eigenvector's components are absent in this expression because of the rotational invariance of the ensemble. From these results, we obtain for the joint probability density of the eigenvalues (in an abuse of notation, in this paper we denote all distribution functions with the same symbol $P)$

$$
P\left(E_{1}, \ldots, E_{N}\right)=Z^{-1} \prod_{i<j=1}^{N}\left|E_{i}-E_{j}\right|^{\beta} \exp \left(-\frac{\beta}{2} \sum_{i=1}^{N} E_{i}^{2}\right),
$$

where $Z$ is a normalization constant.

Interpreting the energies as the (real) positions of some fictitious particles located at $x_{i}=E_{i}$, Eq.(3) may be interpreted as the Boltzmann factor of a classical system, $P\left(x_{1}, \ldots, x_{N}\right)=$ 
$Z^{-1} \exp \left(-\beta V\left(x_{1}, \ldots, x_{N}\right)\right)$, with

$$
V\left(x_{1}, \ldots, x_{N}\right)=-\sum_{i<j} \log \left|x_{i}-x_{j}\right|+\frac{1}{2} \sum_{i} x_{i}^{2} .
$$

The correlations among eigenvalues are therefore described by a two-dimensional Coulomb system made of unit charges (electrons) restricted to move over the real axis. In RMT, the temperature takes the discrete values $\beta=1 / k T=1,2,4$. The harmonic oscillator confining potential is interpreted as the potential produced by a uniform background of opposite charge density. This is therefore a one-dimensional one component plasma $(1 \mathrm{dOCP})$, with particles' interactions as in two dimensions.

The correlations introduced by the Jacobian that are responsible for the Coulomb repulsion among eigenvalues are not a special feature of the particular ensemble of matrices considered above. In fact, for short distances compared to the mean level spacing two neighboring eigenvalues of a generic quantum system repel each other as $\left|E_{+}-E_{-}\right|^{\beta}$. In order to see this, consider the energy surface of two nearby eigenstates $E_{ \pm}$as a function of a set of parameters $\left\{R_{1}, R_{2}, \ldots\right\}$. Two-dimensional degenerate perturbation theory states that in order to produce a degeneracy, $(\beta+1)$ parameters should generically be varied. Moreover, near the degeneracy the contact between the two surfaces has generically the geometry of a diabolical point (a double cone)

$$
E_{ \pm} \propto \pm \sqrt{\sum_{j=1}^{\beta+1} R_{j}^{2}}
$$

It follows from this local geometry that for short distances the probability $p(s)$ to find two neighboring eigenvalues a distance $s=\left|E_{+}-E_{-}\right|$apart from each other is proportional to $s^{\beta}$, as stated above.

From Eqs.(3) and (4) we see that what RMT does is to extend the simple Coulomb interaction which operates at short distances to all levels, located at arbitrary distances. It has moreover been shown [22] that the precise form of the confining potential may modify the density of eigenvalues, but not the local correlations between eigenvalues (at the scale of several mean spacings) that are therefore, in this respect, universal. These universal correlations have been conjectured to describe the local correlations of eigenvalues of fully chaotic systems [23, 4].

The partition function $Z(\beta)=\int \ldots \int d x_{1} \ldots d x_{N} \exp (-\beta V)$ of the $1 \mathrm{dOCP}$ has been computed for any temperature [7]

$$
Z(\beta)=\frac{2 \pi}{\beta^{N[1+\beta(N-1) / 2] / 2}} \frac{\prod_{j=1}^{N} \Gamma(1-\beta j / 2)}{[\Gamma(1+\beta / 2)]^{N}} .
$$

It as also been shown that the minimum of the potential (四) (i.e., the zero temperature configuration) occurs when the particles are located at the zeros of the Hermite polynomials of degree $N$.

At the temperatures $\beta=1,2,4$ required by $\mathrm{RMT}$ all the correlation functions of the gas, $R_{k}\left(x_{1}, \ldots, x_{k}\right)=(N ! /(N-k) !) \int \ldots \int d x_{k+1} \ldots d x_{N} P\left(x_{1}, \ldots, x_{N}\right)$, are known explicitly [7]. For example, for $\beta=2$ and in the large- $N$ limit they take the compact form

$$
R_{k}\left(x_{1}, \ldots, x_{k}\right)=\operatorname{det}\left[\sin \left[\pi\left(x_{i}-x_{j}\right)\right] /\left[\pi\left(x_{i}-x_{j}\right)\right]\right]_{i, j=1, \ldots, k},
$$

while the nearest-neighbor spacing distribution function is given by

$$
p(s)=\frac{32}{\pi^{2}} s^{2} \exp \left(-\frac{4}{\pi} s^{2}\right) .
$$

In fact, this is the $2 \times 2$ Wigner expression, that happens to be a very good approximation to the exact one (which takes a more complicated functional form). For applications of these results and a discussion of their range of applicability in chaotic and disordered systems see for example [田, 司]. 


\section{COMPLEX RANDOM MATRICES}

Here we present an ensemble of random matrices whose (complex) eigenvalues are described by a fully two-dimensional Coulomb gas, as opposed to eigenvalues of random hermitian matrices that lie on a line. Another example of a fully two-dimensional system will be given in next section.

Consider an ensemble $P(H) d H$ of matrices whose matrix elements are independent complex numbers. For simplicity, we consider again a rotationally invariant ensemble

$$
P(H)=\mathcal{N} \exp \left(-\frac{\beta}{2} \operatorname{tr}\left(H H^{+}\right)\right) .
$$

Again, starting from this equation the aim is to write down the joint probability density of the complex eigenvalues $z_{i}, i=1, \ldots, N$. Similar steps as in the previous section leads to [\&]

$$
P\left(z_{1}, \ldots, z_{N}\right)=Z^{-1} \prod_{i<j=1}^{N}\left|z_{i}-z_{j}\right|^{2} \exp \left(-\sum_{i=1}^{N} z_{i}^{2}\right) .
$$

This result is formally identical to the distribution Eq.(3) for $\beta=2$, with the important difference that the eigenvalues now lie in the complex plane. Its thermodynamical interpretation associates to this distribution a 2dOCP (a two-dimensional gas of electrons with a uniform positive background). This analogy has been exploited in Coulomb systems by Jancovici [24].

An explicit formula for the correlations among particles with the distribution (8) can be worked out [B]

$$
R_{k}\left(z_{1}, \ldots, z_{k}\right)=\pi^{-k} \exp \left(-\sum_{i=1}^{k}\left|z_{i}\right|^{2}\right) \operatorname{det}\left[\exp \left(z_{i} \bar{z}_{j}\right)\right]_{i, j=1, \ldots, k} .
$$

For $k=1$ and $k=2$ it reads

$$
\begin{aligned}
& R_{1}(z)=\frac{1}{\pi} \exp \left(-|z|^{2}\right) \sum_{l=0}^{N-1} \frac{|z|^{2 l}}{l !}, \\
& R_{2}\left(z_{1}, z_{2}\right)=1-\exp \left(-\pi\left|z_{1}-z_{2}\right|^{2}\right) .
\end{aligned}
$$

The density of eigenvalues, that has also been studied by Girko [25], tends to a uniform distribution on a disk of radius $|z| \propto \sqrt{N}$, with a Gaussian decay outside it. In the expression of $R_{2}$ the distances are measured in units such that the mean spacing is one.

The nearest neighbor spacing distribution of the two-dimensional gas may be computed from the formula

$$
\int_{0}^{s} p(x) d x=1-\frac{1}{\kappa} \int \ldots \int_{\left|z_{i}\right| \geq s} d^{2} z_{2} \ldots d^{2} z_{N} P\left(z_{1}=0, z_{2}, \ldots, z_{N}\right) .
$$

The normalization constant $\kappa$ is the probability to find one zero at the origin, and the notation is $d^{2} z=d(\operatorname{Re} z) d(\operatorname{Im} z)$. Evaluating explicitly the r.h.s. of Eq.(11) using Eq.(8) one finds [26]

$$
\int_{0}^{s} p(x) d x=1-\prod_{j=1}^{N-1}\left[\mathrm{e}_{j}\left(s^{2}\right) \exp \left(-s^{2}\right)\right],
$$

where $\mathrm{e}_{j}(\alpha)=\sum_{k=0}^{j} \alpha^{k} / k$ ! is the truncated exponential. Deriving this expression with respect to $s$ we get

$$
p(s)=-2 s \prod_{j=1}^{N-1}\left[\frac{\Gamma\left(j+1, s^{2}\right)}{j !}\right] \sum_{k=1}^{N-1}\left[\frac{k \Gamma\left(k, s^{2}\right)}{\Gamma\left(k+1, s^{2}\right)}-1\right],
$$

where $\Gamma(n, x)=\int_{x}^{\infty} \exp (-t) t^{n-1} d t$ is the incomplete gamma function. The integral of this function is one, as required. In order to have also the first moment set to one, one considers the 
normalized distribution $p_{g}(s)=a p(a s)$, with $a=\int_{0}^{\infty} s p(s) d s \approx 1.14293$. The small $s$ expansion of this function is $p_{g}(s)=3.4128 s^{3}+\mathcal{O}\left(s^{5}\right)$.

It is interesting to compare this distribution with the Wigner approximation in two dimensions, which gives excellent results in one dimension (cf Eq.(6)). This is obtained by solving the approximate equation $p(s) d s=\left[1-\int_{0}^{s} p(x) d x\right] s^{3} d s$. Its solution gives the normalized distribution

$$
p_{w}(s)=4 \Gamma(5 / 4)^{4} s^{3} \exp \left(-\Gamma(5 / 4)^{4} s^{4}\right) .
$$

For small spacings, it behaves as $p_{w}(s)=2.7 s^{3}+\mathcal{O}\left(s^{7}\right)$ (see Fig. 1).

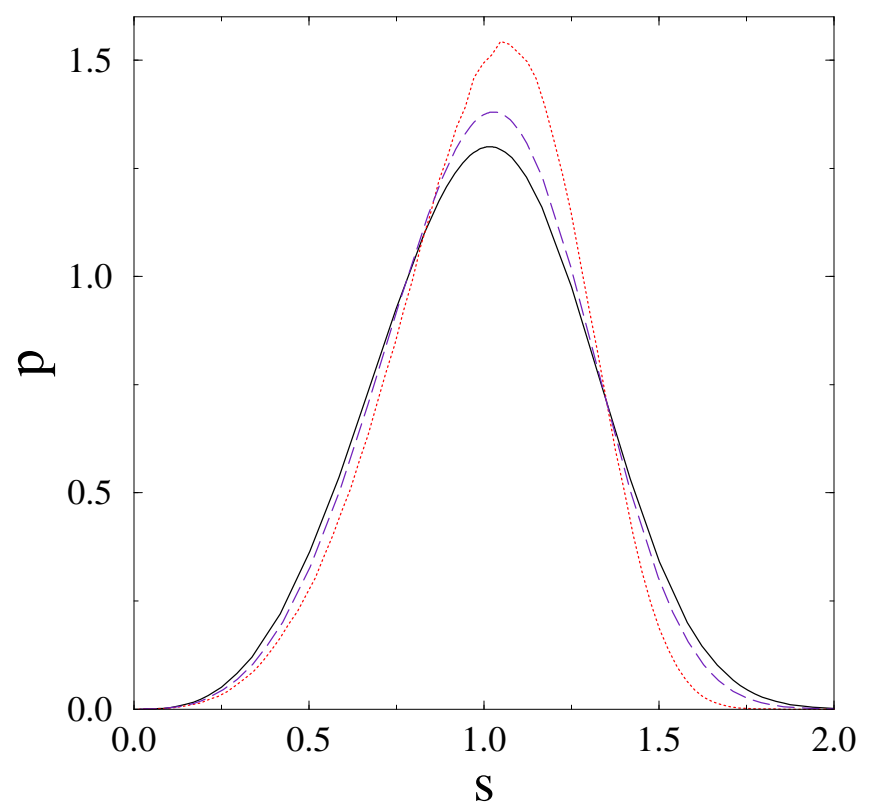

Figure 1: The nearest-neighbor spacing distribution for two-dimensional gases. Full line, $p_{g}$ (Ginibre); dashed line, $p_{w}$ (wigner approximation); dotted line, $p_{r p}$ (zeros of random polynomials).

\section{RANDOM POLYNOMIALS}

As already pointed out, all ensembles of matrices previously considered are rotationally invariant. Since there is no privileged direction in Hilbert space, this induces a very simple form for the probability density $P\left(a_{0}, \ldots, a_{N}\right)$ of the coefficients $\left\{a_{j}\right\}$ of an eigenstate $\psi$ of $H$ expressed in an arbitrary basis

$$
P\left(a_{0}, \ldots, a_{N}\right)=\mathcal{C} \delta\left(\sum_{j=0}^{N}\left|a_{j}\right|^{2}-1\right) .
$$

For convenience, we consider now matrices of dimension $(N+1)$; $\mathcal{C}$ is a normalization constant. We restrict moreover to the case of complex coefficients (systems without time reversal symmetry, $\beta=2$ ).

An interesting way to look at this distribution is the following. Consider the Bargmann (or coherent state) representation, in which the eigenstate takes the form

$$
\psi(z)=\sum_{j=0}^{N} \frac{a_{j}}{\sqrt{j !}} z^{j} .
$$

Because we consider here finite dimensional matrices of size $(N+1)$, the usual series has been 
truncated. The polynomial (14) may be parametrized by its zeros

$$
\psi(z)=\frac{a_{N}}{\sqrt{N !}} \prod_{k=1}^{N}\left(z-z_{k}\right) .
$$

It can easily be shown [27] that the statistical properties of the zeros of a polynomial are unchanged if the distribution (13) is replaced by a Gaussian one

$$
P\left(a_{0}, \ldots, a_{N}\right)=\prod_{i=0}^{N} \frac{1}{2 \pi \sigma_{i}^{2}} \exp \left[-\left|a_{i}\right|^{2} /\left(2 \sigma_{i}^{2}\right)\right] .
$$

The purpose now is, given the probability distribution (15) for the coefficients, to compute the distribution of the zeros of $\psi(z)$ in the complex plane. Many aspects of this problem for different types of polynomials (i.e., having for example some special symmetry, etc) have been understood in recent years [9]-21]. For the particular case (15) of Gaussian independent complex coefficients, all correlation functions have been explicitly computed [10, 12, 13]. The joint probability density is easy to write down. Assuming we absorb the factors $1 / \sqrt{j}$ ! in Eq.(14) into the variance of the coefficients, i.e. we consider polynomials of the form $\psi(z)=\sum_{j=0}^{N} a_{j} z^{j}$, with the $\left\{a_{j}\right\}$ distributed according to Eq.(15). The joint probability density is then [12]

$$
P\left(z_{1}, \ldots, z_{N}\right)=\frac{N !}{\pi^{N} \prod_{k=0}^{N} \sigma_{k}^{2}} \frac{\prod_{i<j}\left|z_{i}-z_{j}\right|^{2}}{\left(\frac{1}{\sigma_{N}^{2}}+\frac{\left|z_{1}+z_{2}+\ldots\right|^{2}}{\sigma_{N-1}^{2}}+\frac{\left|z_{1} z_{2}+z_{1} z_{3}+\ldots\right|^{2}}{\sigma_{N-2}^{2}}+\ldots+\frac{\left|z_{1} z_{2} \ldots z_{N}\right|^{2}}{\sigma_{0}^{2}}\right)^{N+1}} .
$$

Interpreting this distribution as a Boltzmann factor, the numerator corresponds as in previous sections to a two-dimensional Coulomb interaction acting among the zeros. This Coulomb interaction is totally generic for zeros of polynomials with complex coefficients, since it comes from the Jacobian of the transformation and does not depend on the particular distribution assumed for the coefficients. The difference with Ginibre's gas (the 2dOCP) lies in the denominator. The gas (16) is not confined, like the eigenvalues of random Gaussian matrices, by a uniform positive background inducing an harmonic well. The confinement is enforced by a much more complicated many body interaction between the particles. Contrary to the numerator, this term depends on the particular form of the distribution of the coefficients.

In the particular case Eq.(14) where the variances are given by $\sigma_{j}^{2}=1 / j$ !, the density of zeros in the complex plane is [18]

$$
R_{1}(z)=\frac{1}{\pi}\left\{1-g\left(|z|^{2}\right)\left[1+N-|z|^{2}+|z|^{2} g\left(|z|^{2}\right)\right]\right\}
$$

where $g(x)=\left(x^{N} / N !\right) / \mathrm{e}_{N}(x)$. As for the eigenvalues of complex matrices, in the large- $N$ limit this density is uniform on a disk of radius $\propto \sqrt{N}$. Outside the disk, it tends to zero much slower than the $2 \mathrm{dOCP}$, since for large radius the tail of the density decays as $1 /|z|^{4}$.

A general expression for the two-point correlation function has been derived by Hannay [13]. For large $N$ and in the uniform-density region it is given by

$$
R_{2}\left(z_{1}, z_{2}\right)=\frac{\left[\left(\sinh ^{2} v+v^{2}\right) \cosh v-2 v \sinh v\right]}{\sinh ^{3} v},
$$

with $v=\pi\left|z_{1}-z_{2}\right|^{2} / 2$ (here again we measure the distances in units such that the mean spacing is one).

A detailed comparison between the random-polynomial gas and the $2 \mathrm{dOCP}$ has been made by Forrester and Honner [21]. They found that the first moment $\int d^{2} z\left[R_{2}(z, 0)-1\right]=-1$ in both cases, which means that a perfect screening is operating. However, the second moment differs, $\int d^{2} z\left|z^{2}\right|\left[R_{2}(z, 0)-1\right]=-1 / \pi$ for the $2 \mathrm{dOCP}$ while it vanishes for the random polynomial gas. 
In the case of the $2 \mathrm{dOCP}$, this result is known as the Stillinger-Lovett sum rule. The physical interpretation of the result obtained for the zeros of random polynomials is not yet clear.

The nearest-neighbor spacing distribution for the gas of zeros follows from Eqs.(11) and (16). We have not been able to compute the integral. However, it may be of some practical interest to note that the numerical curve of $p(s)$ for zeros of random polynomials (in the constant density region) is well fitted by the expression $p_{r p}(s)=2.211 s^{3} \exp \left(-0.389 s^{5.52663}\right)$ (see Fig. 1).

The validity of these results in the study of the structure of eigenstates of classically chaotic systems has been investigated in [12, 14, 15].

\section{CONCLUDING REMARKS}

This brief survey emphasizes the strong connection existing between on the one hand quantum statistical theories like random matrix theory or random polynomials and the physics of classical Coulomb systems on the other. The benefits are mutual. The thermodynamical interpretation serves as a guideline in the quantum mechanical studies. In the opposite direction, this connection is a powerful tool to compute analytically the correlation functions of the classical gas, at least at certain temperatures.

Many open problems lie at this interface. One example is the gas associated to the zeros of random polynomials, whose relevance and physical interpretation is still unclear from the thermodynamical point of view. This gas contains, aside a Coulomb repulsion, many body interactions among the particles. These interactions confine the system (they compensate the strong Coulomb repulsion) and also modify the correlations and the local response of the gas. Nothing is known about its behavior for temperatures different from $\beta=2$.

Another example concerns the fact that all the standard ensembles of random matrices or polynomials are related to some classical interacting gas with particles carrying the same charge (with eventually a compensating uniform background). It would be interesting to study ensembles of matrices or polynomials associated to two (or multi) component plasmas, whose physical behavior is known to be much richer.

\section{Acknowledgments}

Le Laboratoire de Physique Théorique et Modèles Statistiques est une Unité de recherche de l'Université de Paris XI associée au CNRS.

\section{References}

[1] E. P. Wigner, Proc. Cambridge Philos. Soc. 47 (1951) 790. See also Ref.[3].

[2] F. Dyson, J. Math. Phys. 13, 90 (1972). See also Ref.[3].

[3] C. E. Porter (Ed.), Statistical Theories of Spectra: Fluctuations, Academic Press, New York, 1965.

[4] Proceedings of the Les Houches Summer School, Chaos and Quantum Physics, Les Houches, Session LII (M.-J. Giannoni, A. Voros and J. Zinn-Justin eds.), Elsevier 1991.

[5] Proceedings of the Les Houches Summer School, Mesoscopic Quantum Physics, Les Houches, Session LXI (E. Akkermans, G. Montambaux, J.-L. Pichard and J. Zinn-Justin eds.), Elsevier 1995.

[6] A. M. Odlyzko, The $10^{20}$-th zero of the Riemann zeta function and 175 million of its neighbors, AT \& T Report, 1992 (unpublished).

[7] M.L. Mehta, Random Matrices, Academic Press, New York, 1991.

[8] J. Ginibre, J. Math. Phys. 6, 440 (1965). 
[9] A.T. Bharucha-Reid and M. Sambandham, Random Polynomials, Academic Press, 1986.

[10] E. Bogomolny, O. Bohigas and P. Lebœuf, Phys. Rev. Lett. 68 (1992) 2726.

[11] A. Edelman and E. Kostlan, Bull. Amer. Math. Soc. 32 (1995) 1.

[12] E. Bogomolny, O. Bohigas and P. Lebœuf, J. Stat. Phys. 85 (1996) 639.

[13] J. Hannay, J. Phys. A: Math. Gen. 29 (1996) L101.

[14] P. Leboeuf and P. Shukla, J. Phys. A: Math. Gen. 29 (1996) 4827.

[15] T. Prosen, J. Phys. A 29 (1996) 4417; Physica D 91 (1996).

[16] P. Bleher and X. Di, J. Stat. Phys. 88 (1997) 269.

[17] J. Hannay, J. Phys. A: Math. Gen. 31 (1998) L755.

[18] P. Leboeuf, J. Stat. Phys. 95 (1999) 651.

[19] B. Shiffman and S. Zelditch, Distribution of zeros of random and quantum chaotic sections of positive line bundles, preprint math/9803052; P. Bleher, B. Shiffman and S. Zelditch, Universality and scaling of correlations between zeros on complex manifolds, preprint 1999.

[20] S. Nonnenmacher and A. Voros, J. Stat. Phys. 92 (1998) 431.

[21] P. Forrester and G. Honner, Exact statistical properties of the zeros of complex random polynomials, to appear in J. Stat. Phys.

[22] E. Brézin and A. Zee, Nucl. Phys. B402 (1993) 613.

[23] O. Bohigas, M.-J. Giannoni and C. Schmit, Phys. Rev. Lett. 52 (1984) 1.

[24] B. Jancovici, Phys. Rev. Lett. 46, 386 (1981).

[25] V. L. Girko, Theory Prob. Appl. 22 (1977) 156.

[26] R. Grobe, F. Haake and H. J. Sommers, Phys. Rev. Lett. 61 (1988) 1899.

[27] M. Kac, Probability and Related Topics in Physical Sciences, Wiley, New York, 1959. 